\title{
COVID-19 and Minimizing Micro-Spatial Interactions
}

\author{
SUSAN BURTNER and ALAN T. MURRAY, Department of Geography, University of California \\ at Santa Barbara, Santa Barbara, CA, 93106, USA
}

COVID-19, the novel coronavirus that has disrupted lives around the world, continues to challenge how humans interact in public and shared environments. Repopulating the micro-spatial setting of an office building, with virus spread and transmission mitigation measures, is critical for a return to normalcy. Advice from public health experts, such as maintaining physical distancing from others and well-ventilated spaces, are essential, yet there is a lack of sound guidance on configuring office usage that allows for a safe return of workers. This paper highlights the potential for decision-making and planning insights through location analytics, particularly within an office setting. Proposed is a spatial analytic framework addressing the need for physical distancing and limiting worker interaction, supported by geographic information systems, network science, and spatial optimization. The developed modeling approach addresses dispersion of assigned office spaces as well as associated movement within the office environment. This can be used to support the design and utilization of offices in a manner that minimizes the risk of COVID-19 transmission. Our proposed model produces two main findings: (1) that the consideration of minimizing potential interaction as an objective has implications for the safety of work environments, and (2) that current social distancing measures may be inadequate within office settings. Our results show that leveraging exploratory spatial data analyses through the integration of geographic information systems, network science, and spatial optimization, enables the identification of workspace allocation alternatives in support of office repopulation efforts.

CCS Concepts: • Applied computing $\rightarrow$ Multi-criterion optimization and decision-making; $\bullet$ Mathematics of computing $\rightarrow$ Integer programming; • Information systems $\rightarrow$ Data analytics; Geographic information systems;

Additional Key Words and Phrases: Spatial optimization, exploratory spatial data analysis, network science, spatial interaction, health informatics

\section{ACM Reference format:}

Susan Burtner and Alan T. Murray. 2022. COVID-19 and Minimizing Micro-Spatial Interactions. ACM Trans. Spatial Algorithms Syst. 8, 3, Article 18 (January 2022), 17 pages.

https://doi.org/10.1145/3486970

This work was supported in part by a Multidisciplinary Research on the Coronavirus and its Impacts (MRCI) grant, awarded from the Graduate Division of the University of California at Santa Barbara.

Authors' address: S. Burtner and A. T. Murray, Department of Geography, 1832 Ellison Hall, UC Santa Barbara, CA, USA, 93106-4060; emails: \{sab00, amurray\}@ucsb.edu.

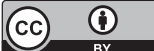

This work is licensed under a Creative Commons Attribution International 4.0 License.

(c) 2022 Copyright held by the owner/author(s).

2374-0353/2022/01-ART18

https://doi.org/10.1145/3486970
}

ACM Transactions on Spatial Algorithms and Systems, Vol. 8, No. 3, Article 18. Publication date: January 2022. 


\section{INTRODUCTION}

The novel coronavirus COVID-19 has unequivocally altered the lives of Americans. The United States presents a particularly vexing policy landscape as states, counties, and localities implement different measures to battle the virus. Combined with an array of misinformation and falsehoods at the highest levels of government, the United States lagged behind many other countries in its virus mitigation efforts in 2020 and hundreds of thousands of lives were lost. While U.S. Federal Drug Administration approval and growing distribution of vaccines lends hope to seeing an end to the pandemic, most American adults are not projected to receive a vaccine until late summer or early fall of 2021 [McNeil 2020; Cunningham and Ellerbeck 2021].

As Americans continue to seek out a return to normalcy, including working at the office, it is clear that established mitigation measures to curb virus transmission will remain critical over the coming months, especially if many new variants of the coronavirus emerge. Some recurrent measures are the practice of social (or physical) distancing to maintain at least six feet between other people outside of one's household, wearing a facial covering, and regularly washing one's hands. These mitigation efforts have been cited by public health experts as key for preventing the spread of COVID-19 [Centers for Disease Control and Prevention 2021]. What is missing from the planning of increased work activity, however, is better guidance on how companies, organizations, and individuals should incorporate physical distancing into shared spaces. Many employers are currently allowing a majority of their employees to work from home, but the transition back to shared micro-spatial environments such as offices is in need of detailed systematic study and planning.

Offering much potential to support efforts to safely return employees to the workplace are location analytics, including spatial optimization. Such approaches provide insight for exhaustive consideration of potential worker micro-spatial configurations, moving away from ad hoc decisionmaking, in order to enhance worker safety. For many, the primary objective for transitionalpandemic work environments will be to safely increase the number of people that may resume use of their offices yet keep interaction and potential for virus spread in check. In the following sections, we explore how location analytics, particularly a dispersion-based spatial optimization model, can be utilized to help plan and guide office repopulation efforts in a micro-spatial office environment. Our proposed model and analysis show that differing objectives are possible in office repopulation, suggesting important alternatives that should be considered. Our results also identify two main findings: (1) that considering the potential for interaction between individuals results in a safer spatial configuration of dispersed offices, and (2) that even a more restrictive social distance of 9 feet [Setti et al. 2020] between individuals is problematic and may create too many opportunities for close contact in an office environment. The hope is that our methods and findings help to guide stakeholders in their organizational management, particularly in the context of virus spread mitigation.

\section{BACKGROUND}

Location analytics, and spatial optimization and location models in particular, often deal with prescriptive planning and decision-making that involves geographic placement and allocation of resources. A traditional area of focus is associated with the siting of service facilities in such a manner that the costs of distributing goods is minimized in order to meet demand requirements [Church and Murray 2009]. Equally important in many cases are efforts to maximize accessibility as well as provide service coverage. There are many situations in which service facilities or activities must be dispersed, particularly in the case of hazardous exposure and/or obnoxious service impacts. Examples include services that produce odors, noises, chemical byproducts, etc., such as waste disposal sites, airports, fire stations, fuel processing, munitions storage and the like, or any activities that would expose communities to pollutants. The prevailing attitude is that such 
services, goods, and activities are needed in a community, but "not in my backyard" [Kunreuther et al. 1993]. Accordingly, the intent is that noxious facilities should be placed far away from people, or in many cases away from each other [Murray et al. 1998].

Most certainly a concentration of hazardous materials or obnoxious services in any one area is not beneficial and could in many cases represent an elevated level of danger to localities. To support mitigation efforts, a range of dispersion models have been developed for planning, management, and decision-making [Erkut and Neuman 1991; Niblett and Church 2015; Church and Murray 2018]. Two fundamental approaches are anti-covering and $p$-dispersion models. The anticovering location problem (ACLP) establishes a restriction-based standard such that a minimum distance between sited facilities must be maintained [Moon and Chaudhry 1984; Church and Murray 2009]. The ACLP has been utilized in many planning contexts, including retail system design [Zeller et al. 1980], forest and ecological management [Church et al. 1998; Murray 1999; Downs et al. 2008], and convicted criminal residency restrictions [Grubesic and Murray 2008]. Another approach is the $p$-dispersion problem, seeking to maximize the distance between sited facilities [Kuby 1987]. Church and Murray [2018] demonstrated that the $p$-dispersion problem could be structured as an equivalent ACLP. This suggests that the ACLP is a more generalizable approach to account for an array of dispersion situations.

The need to disperse activities is well recognized in facility siting and layout design, and network frameworks have proven beneficial in addressing constrained spatial arrangements. Classic network optimization problems, such as finding the shortest path to a destination, the transportation problem, and general assignment problems, have leveraged network structures in route planning [Church and Murray 2009; Newman 2018]. Many potentially hazardous processes, such as chemical or other manufacturing systems, exist within a network structure where processing units, equipment, and procedures must happen in sequence and within a set distance of one another [American Institute of Chemical Engineers 2018]. Facility siting usually transpires at a large network scale where the broader supply chain, distance to relevant and vulnerable populations, and service demand must be considered, while concerns over facility layout involve more of the internal physical arrangement of equipment and production spaces. When considering modeling possibilities, many facility layout problems can be cast as combinatorial optimization problems, aided by a range of software packages [Meller and Gau 1996]. Location problems involving facilities such as offices can be captured through the use of binary assignment or mixed-integer programs that use network representations to reflect the spatial relationships between places [ReVelle 1993; Erkut et al. 1996].

Recent research has sought to address the pressing need of spatial allocation and selection during a pandemic. Murray [2020] applied the ACLP to the classroom setting to ensure social distancing, while Kudela [2020] modeled social distancing as a p-dispersion problem. Kudela [2020] compared the computational implications of alternative discretized space representations, while Murray [2020] demonstrated how social distance standards could be considered, highlighting that various seating arrangements alternatives are possible through different restriction separation distances ( $r$-separation distances) but that accommodating the most individuals possible will not happen by chance. The present work relies on exploration through restriction or $r$-separation distance manipulation but seeks to move beyond the classroom setting to examine a similar micro-spatial environment where individual interaction must be accounted for over the course of a day.

We define a micro-spatial environment as one in which human movement is limited, such as within the floor of a building. In the case of COVID-19 planning for an office setting, certain movements must be anticipated and mitigated to the greatest extent possible. Specifically, there is a desire to accommodate the most workers possible in a building. However, this must be done according to mitigation efforts and safety constraints. Minimizing the potential for spatial interaction necessarily suggests the fewest workers possible, dispersed to the greatest extent. These 
opposing objectives of repopulation imply starkly different spatial arrangements of workers. It is therefore critical to have a holistic approach to help identify a range of potential scenarios, enabling the selection of plans to best suit the needs of individual companies and organizations as well as unique working environments. Accordingly, multiple objective modeling very much reflects such a planning and decision-making approach. ReVelle [1993], Medrano and Church [2015], and Wei and Murray [2018] highlight both the challenges and utility of multiple objective considerations.

\section{CASE STUDY}

Micro-spatial environments present a new and unique challenge for planning and management of shared spaces during a pandemic. The reasons for this are due to spatial uniqueness in both layout and resulting movement patterns. This includes the location and ventilation of individual offices and workspaces, but also entry and exits as well as restrooms, drinking fountains, and other shared amenities. These unique micro-scale environments along with social distancing restrictions highlight that detailed individual analysis must be carried out to identify and select from among potential mitigation alternatives. As a representative example, a building on the campus of the University of California, Santa Barbara serves as a case study. The building is Ellison Hall, and the analysis focuses on the fourth floor of the building.

The University of California, Santa Barbara is home to around 26,000 students, divided among 23,000 undergraduate students and around 3,000 graduate students. There are about 1,100 faculty, 300 postdoctoral fellows, and around 4,000 staff members. Ellison Hall is one of the older buildings on campus; it has six floors which are the primary home to the departments of Geography, Political Science, and the Earth Research Institute, constituting a fair mix of offices, classrooms, laboratories, and conference rooms. The Geography department occupies a large portion of Ellison Hall, taking up nearly the entirety of four of the six floors. The department has 28 faculty, around 100 graduate students (though not all are in residence), around 15 professional researchers and postdoctoral scholars, and 11 staff members. As shown in Figure 1, office spaces on the fourth floor of Ellison Hall vary in size, and spatial patterns of movement and mobility necessarily change over the course of a day.

Effectively dealing with physical movements within a building requires an understanding of specific work and social dynamics as well as how the physical structure affords interaction. COVID-19, however, requires a reexamination of social interactions, and a transitional post-COVID-19 work environment may severely limit access to other individuals and spaces of congregation. In this case study, we incorporate stipulations oriented toward curbing social behaviors in an effort to reduce interaction and virus transmission. Many of these stipulations are based on rules already in place for Ellison Hall, and others are associated with a reimagined work environment of greater population density. In accordance with county health guidelines, the entire building can have no more than $15 \%$ occupancy, and this constraint applies to each floor of Ellison Hall. ${ }^{1}$ For simplicity, we assume that once someone enters a building, they do not exit until they are ready to leave. Current restrictions permit only one occupant in an assigned office at any time to reduce the amount of shared air. Other protocols apply as well, including limits of only one person in stairwells (which connect the building entrances and exits to floor entrances and exits), restrooms, or the lounge at a time. According to stricter social distancing guidelines set forth by the university's Office of Research ${ }^{2}$ and emerging research that even 6 feet of social distancing is not enough

\footnotetext{
${ }^{1}$ The building occupancy of $15 \%$ was set in place for the fall quarter of 2020 , but has since increased to $25 \%$ as of February 2021, and will likely increase as more individuals become vaccinated.

${ }^{2}$ This is sourced from an internal memo circulated to building committees. A similar, publicly available resource is available here: http://microfluidics.cnsi.ucsb.edu/wiki/lib/exe/fetch.php?media=wiki:covid-19_risk_mitigation_presentation.pdf.
} 


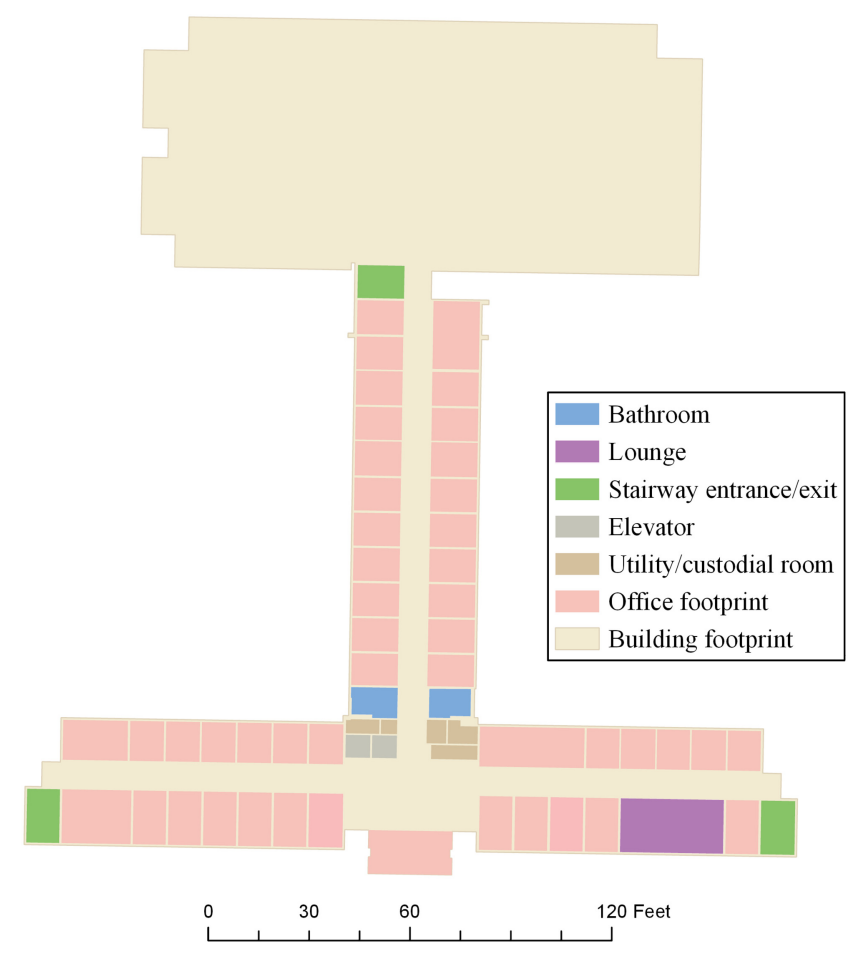

Fig. 1. Spatial layout of the fourth floor of Ellison Hall.

[Setti et al. 2020], individuals should maintain at least 9 feet of distance between others and remain in their assigned office with doors closed, only going to the restroom or lounge when necessary.

Based on these safety protocols, no two neighboring offices can simultaneously be assigned or occupied. Due to airflow and other considerations, such a restriction standard mitigates the potential for disease spread. Of course, movement within the floor represents another aspect of risk, so offices near high use areas have increased vulnerability as does the potential of travel path overlap between two or more individuals. The goal therefore is to represent the various features of management goals in a spatial analytic model in order to identify office occupancy plans that enable workers to return as safely as possible.

\section{METHODS}

The stipulations mentioned previously present a candidate set of situational modifications to a work environment. This section details how these issues can be addressed using a combination of geographic information systems (GIS), network science, spatial optimization, and multiobjective modeling structured to facilitate analysis, planning, and decision-making. An overview of a framework to support micro-spatial office space planning to mitigate virus spread is structured in Figure 2. Each of the constituent components are now discussed in terms of their contribution to the broader framework.

\subsection{Data Collection \& Processing}

The need for data collection, creation, management and cleaning reflected in Figure 2 are always present and a prerequisite to modeling and analysis. Planning to allocate office usage with respect to any type of distance consideration must involve the ability to derive distances between offices 


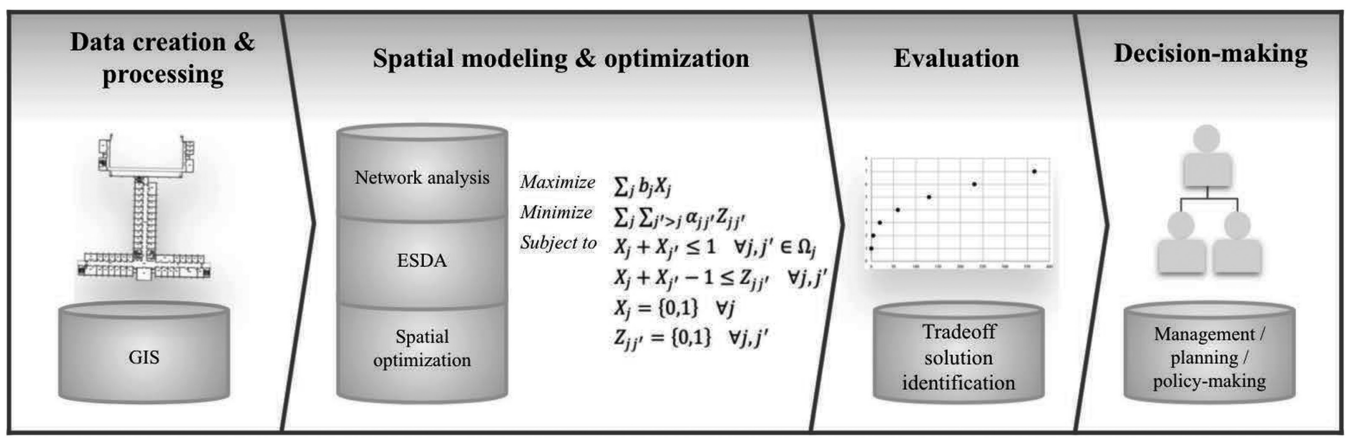

Fig. 2. Overview of methodological framework.

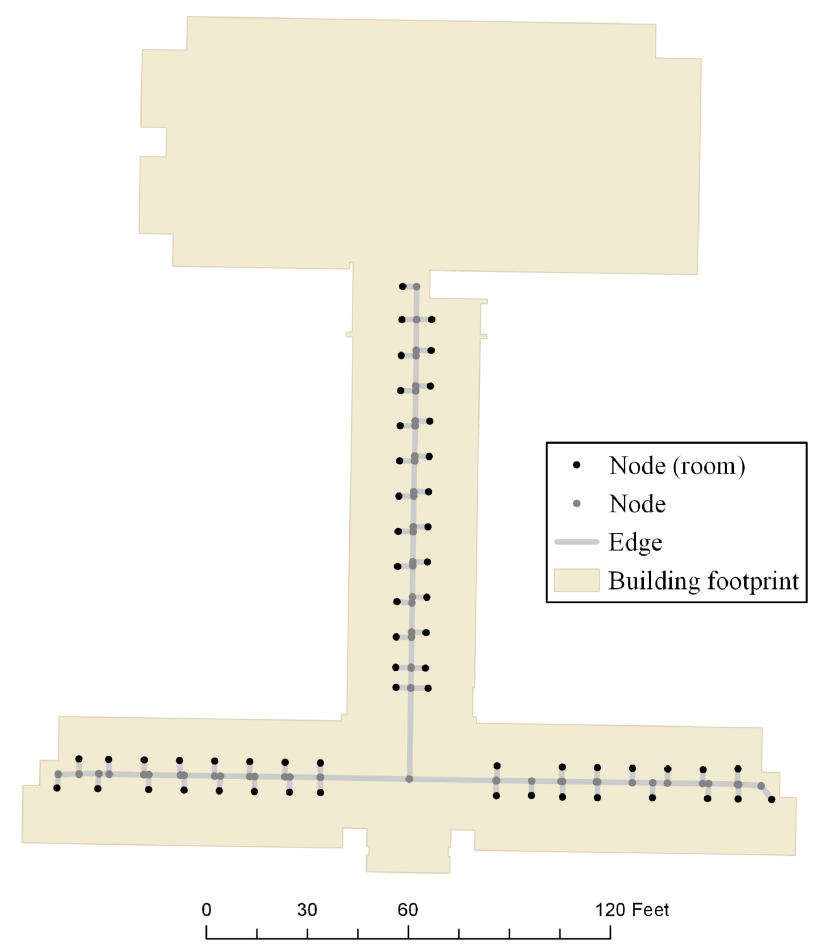

Fig. 3. Network of feasible walking paths and destinations for the fourth floor of Ellison Hall.

and other relevant interior amenities, such as bathrooms and the lounge, along a network of feasible walking paths. Constructing a network can be accomplished using GIS through the use of digitized building footprints. The technical details of doing this are beyond the focus of the paper, but a derived network for the fourth floor of Ellison Hall based on rooms/stairway doorways and entrances is shown in Figure 3. Hallways are generalized to centerlines (edges) and room designations (nodes) correspond to doors as well as edge intersections.

\subsection{Spatial Modeling \& Optimization}

A number of elements associated with spatial modeling and optimization are indicated in Figure 2, including network analysis, exploratory spatial data analysis (ESDA), and spatial optimization. 
Beyond data creation and processing, GIS also serves as a valuable tool for network analysis, ESDA, and deriving walking path overlap within the building. The latter reflects the utilized measure for potential office interaction relied upon here. There are a range of methods to support various aspects of network analysis. Central to our analysis is the calculation of shortest paths through the network, from offices to facilities, and from which other forms of analysis such as calculating path overlap are possible. The overlap of paths between offices and facilities is based on the topological overlay of the union of two different offices' shortest paths to designated facilities. Topological overlay, and specifically geometric intersection, is a prominent generalization tool in GIS, but is implemented less often for overlapping lines whose intersection forms a line segment. To more precisely specify this, further mathematical clarification is provided. We define overlap $\alpha_{j j^{\prime}}$ between two offices $j$ and $j^{\prime}$ (with associated facilities) to include all $x y$ points along the line segment $\overline{x y}$ such that:

$$
\alpha_{j j^{\prime}}=\left\{\overline{x y}: x y \in\left(L_{j 0} \cup L_{j 1}\right) \cap\left(L_{j^{\prime} 0} \cup L_{j^{\prime} 1}\right)\right\}
$$

where $L_{j k}$ is the line-based path from office $j$ to facility $k$ (here $k \in\{0=$ bathroom, $1=$ lounge $\}$ ). As the number of accessible facilities increases, $k$ can be generalized (e.g., $0,1,2, \ldots, n$ ) and the measure of path overlap becomes:

$$
\alpha_{j j^{\prime}}=\left\{\overline{x y}: x y \in\left(L_{j 0} \cup L_{j 1} \ldots \cup L_{j n}\right) \cap\left(L_{j^{\prime} 0} \cup L_{j^{\prime} 1} \ldots \cup L_{j^{\prime} n}\right)\right\}
$$

The basics of ESDA involve the exploration of spatial data through the use of quantitative methods, particularly supported by visualization approaches [Anselin and Bao 1997]. As one of the relevant building rules is preventing adjacent offices from being simultaneously used or selected, ESDA in the form of spatial statistical and network methods allowed for the visualization and analysis of the distances at which offices could or could not be sited. ESDA within a GIS furthermore provided a way to validate different scenario parameters and plan development.

As mentioned previously, building managers may have different objectives for building occupancy during COVID-19. Maintaining physical distancing and reducing the potential for interaction between individuals are major but contradictory goals in office space repopulation. These conflicting aims can be addressed through the development and application of a bi-objective mathematical program, where the goal is to maximize building or floor occupancy while minimizing the potential for interaction. Consider the following notation:

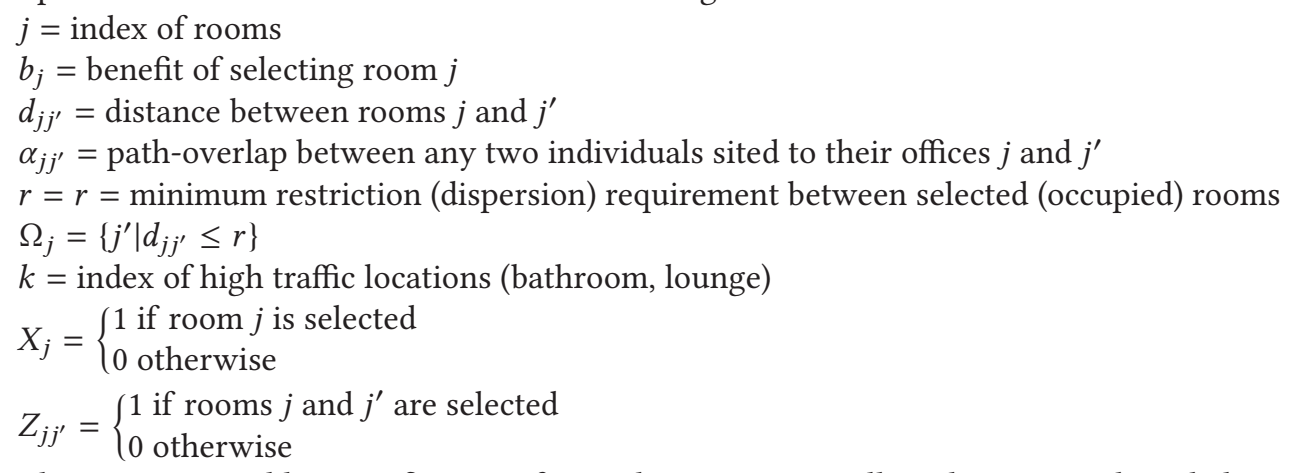

This notation enables specification of spatial structure as well as planning goals and objectives. The distance along the network between two offices $j$ and $j^{\prime}$ is given by $d_{j j^{\prime}}$. Spatial structure in the form of $\Omega_{j}$ indicates the set of all neighboring offices $j^{\prime}$ that are within (less than) some minimum separation distance $\mathrm{r}$ of a given office $j$. Interaction is captured by the amount of path-overlap between any two individuals sited to their offices $j$ and $j^{\prime}$ across all walking paths to permissible facilities, $\alpha_{j j^{\prime}}$. Primary decision variables are $X_{j}$, indicating which rooms are to be repopulated. 
Additionally, decision variables $Z_{j j^{\prime}}$ are necessary to track potential interaction of individuals in two selected offices, $j$ and $j^{\prime}$.

With this notation, an optimization model that simultaneously addresses physical distancing and seeks to minimize the potential for behavioral interaction can be derived. The formulation of this bi-objective mathematical program, which we specify as path-overlap, is as follows:

$$
\begin{gathered}
\text { Maximize } \sum_{j} b_{j} X_{j} \\
\text { Minimize } \sum_{j} \sum_{j^{\prime}>j} \alpha_{j j^{\prime}} Z_{j j^{\prime}} \\
\text { Subject to } X_{j}+X_{j^{\prime}} \leq 1 \forall j, j^{\prime} \in \Omega_{j} \\
X_{j}+X_{j^{\prime}}-1 \leq Z_{j j^{\prime}} \forall j, j^{\prime} \\
X_{j}=\{0,1\} \forall j \\
Z_{j j^{\prime}}=\{0,1\} \forall j, j^{\prime}
\end{gathered}
$$

Objective (3) maximizes the number of offices sited, while objective (4) minimizes total path overlaps between two sited offices, only counting the distance of the overlap once in the summation. Constraints (5) ensure that at most one office can be selected for any pair of adjacent rooms. Constraints (6) track when two rooms have both been selected. Binary requirements are imposed in constraints (7) and (8).

To further illustrate features of the model, suppose room $j$ is selected, meaning that $X_{j}=1$. Given $X_{j}+X_{j^{\prime}} \leq 1$ in (5), this implies $1+X_{j^{\prime}} \leq 1$. Thus, $X_{j^{\prime}}$ must equal 0 for all $j^{\prime} \in \Omega_{j}$. That is, any rooms that are within the restriction distance $r$ defining the set $\Omega_{j}$ are expressly prohibited from simultaneous selection. Conversely, if room $j$ is not selected, then $X_{j}=0$ and $0+X_{j^{\prime}} \leq 1$. Thus, selection is not restricted, so $X_{j^{\prime}}$ may equal 0 or 1 for all $j^{\prime} \in \Omega_{j}$. The implication of constraints (5) is that at any point in time, at most one room within an adjacent pair of rooms can be occupied, as intended.

The second set of constraints, (6), serve to track pairs of selected rooms. The model must therefore account for when two offices are selected, represented by $Z_{j j^{\prime}}=1$. Three cases are possible. Given $X_{j}+X_{j^{\prime}}-1 \leq Z_{j j^{\prime}}$ in (6), the first case is that both rooms are selected $\left(X_{j}=X_{j^{\prime}}=1\right)$. This implies $1+1-1 \leq Z_{j j^{\prime}}$, or $1 \leq Z_{j j^{\prime}}$. Thus, the constraint forces $Z_{j j^{\prime}}$ to be 1 , accurately indicating that both offices are selected. The second case is when one room is selected but the other is not (e.g., $X_{j}=1$ and $X_{j^{\prime}}=0$ ). This implies $1+0-1 \leq Z_{j j^{\prime}}$, or $0 \leq Z_{j j^{\prime}}$. Given the orientation of objective (4), the value of this decision variable $Z_{j j^{\prime}}$ will equal 0 . Again, this accurately reflects the intended outcome to only track when both rooms have been selected. The final case is when both rooms are not selected (e.g., $X_{j}=X_{j^{\prime}}=0$ ). This implies $0+0-1 \leq Z_{j j^{\prime}}$, or $-1 \leq Z_{j j^{\prime}}$. Given the binary constraint (8) for $Z_{j j^{\prime}}$ combined with objective (4), the value of $Z_{j j^{\prime}}$ will equal 0 .

Collectively then, constraints (5) and (6) combined with objectives (3) and (4) reflect the intentions of planning efforts to select rooms that adhere to physical distancing requirements as well as track potential interaction between sited office pairs.

\subsection{Evaluation}

Highlighted in Figure 2 is that evaluation is an important process in the overall analysis effort. The developed the spatial optimization model to address physical distancing and potential interaction is bi-objective, reflecting the need to simultaneously consider two concerns. It is worth pointing 
out that if objective (4) is ignored, the model reduces to a special case of the ACLP as only physical distancing would be imposed. Conversely, ignoring objective (3) gives a model focused on minimizing potential interaction, with an assurance that physical distancing is maintained.

Multi-objective optimization problems represent a decision-making environment where there are potentially many different optimal solutions, or Pareto tradeoffs. Identifying these solutions can be challenging and has been approached in two basic ways: the weighting method and the constraint method [Cohon 1978]. While other techniques exist, the weighting method is relied upon here to identify Pareto tradeoffs. Suppose that $o_{1}=\sum_{j} b_{j} X_{j}$, (3), and $o_{2}=\sum_{j} \sum_{j^{\prime}>j} \alpha_{j j^{\prime}} Z_{j j^{\prime}}$, (4). Then, the weighting method consists of the use of a parameter, $w$, to scale one objective against the other. This allows for both objectives to be incorporated into one expression, allowing solutions to be calculated using commercial integer programming software. Specifically, this is done as follows:

$$
\text { Maximize } w_{1}-(1-w) o_{2}
$$

where $w \in[0,1]$

Objective (9) reflects that $(1-w) o_{2}$ is subtracted from $w o_{1}$ since objective (4) has a minimization orientation. A potential issue to consider for integer programming models is that the weighting method may not be able to identify all Pareto tradeoffs [see Medrano and Church 2015]. Accordingly, the constraint method represents a potential alternative for finding the Pareto tradeoffs [ReVelle 1993; Wei and Murray 2018]. Application of the weighting method requires an enumeration of different values of $w$, then solution and evaluation of each resulting problem instance.

\subsection{Decision-making}

The purpose of any spatial optimization model is to provide information pertinent to decisionmaking, but it is not necessarily a straightforward process. Location analytics recognize descriptive and prescriptive contexts, and spatial optimization models are used in both [Church and Murray 2009]. In support of room/office repopulation efforts, prescriptive allocations of workers are sought. Once solutions are generated, stakeholders such as building managers may evaluate given solutions against additional considerations. This is reflected in the decision-making component of Figure 2. The field of utility theory is one area devoted to the study of how individuals identify preferences among many alternatives, and the potential models that can preserve the ordering of such alternatives [Fishburn 1970]. Other techniques such as decision analysis, risk analysis and analytic hierarchy process offer either qualitative or quantitative evaluations of risk and decision possibilities [Zahedi 1986; Balson et al. 1992]. Across each of these techniques, numerical measures such as eigenvalues, individually defined weights or probabilities, are used to differentiate between alternatives and summarize the structural properties of differences among possible scenarios, aiding management and informing broad policy decisions. The framework in Figure 2 therefore recognizes interactive components that are relied upon in concert to derive plans and may include complex approaches to support decision-making.

\section{APPLICATION RESULTS}

As noted previously, the case study looks at the fourth floor of Ellison Hall on the University of California, Santa Barbara campus. The fourth floor of Ellison Hall sees a lot of foot traffic in that it has 46 offices, three entries/exits, bathrooms for two genders, and a lounge for storing and preparing food, as shown in Figure 1. There are also two elevators and a drinking fountain, but these are generally prohibited from use under pandemic conditions. As mentioned previously, of primary interest in planning efforts are ensuring safety and avoiding potential virus transmission. Thus, the locations of occupied/assigned offices with respect to one another, the lounge, and the 


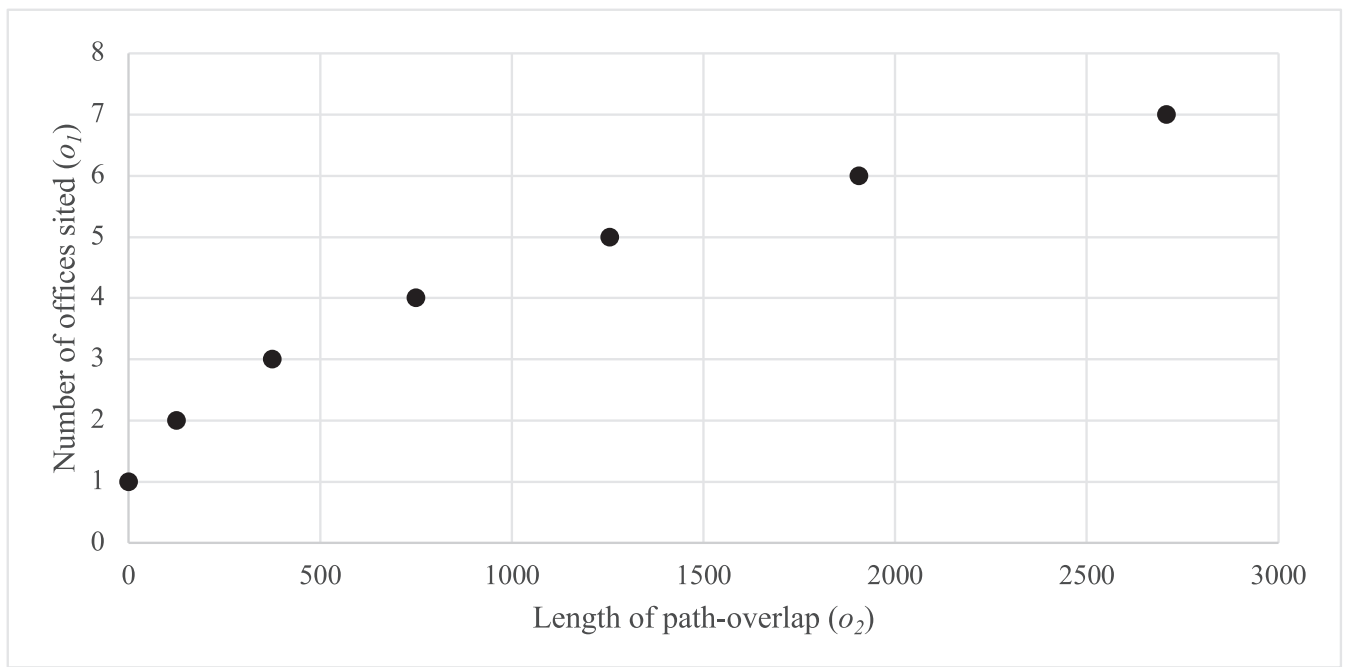

Fig. 4. Pareto tradeoffs for $r=40.36 \mathrm{ft}$.

Table 1. Pareto Tradeoffs for $r=40.36 \mathrm{ft}$

\begin{tabular}{lcc}
\hline $\boldsymbol{w}$ & Number of offices, (3) & Path-overlap, (4) \\
\hline 0.0010 & 1 & 0.0 \\
0.9930 & 2 & 124.91 \\
0.9970 & 3 & 374.73 \\
0.9980 & 4 & 749.47 \\
0.9981 & 5 & $1,255.03$ \\
0.9985 & 6 & $1,905.96$ \\
0.9988 & 7 & $2,707.65$ \\
\hline
\end{tabular}

bathroom are a major concern. The shortest network distance from each office to one another, the lounge and the bathroom were derived in a Jupyter notebook (Python) environment and used in calculating path-overlap (1) for each pair of offices.

ESDA was also conducted using Python and ArcGIS Pro to determine the spatial distance at which all of the building management's spatial conditions were met. The spatial distances between offices in our case study range from around 10 to about 253 feet. An $r$-separation distance of $r=$ 40.46 feet was determined to be the minimum distance which meets social distancing requirements, the ventilation condition that no two adjacent offices are sited next to one another, and a building occupancy requirement of $15 \%$.

The path-overlap model was implemented and solved using FICO॰ Xpress within the Jupyter notebook environment, run on a personal laptop computer (a Macintosh with $2.3 \mathrm{GHz}$ Quad-Core Intel Core i7 processor and 16 GB RAM). The Pareto tradeoffs were found using the weighting method, where arrays of the weight $w \in[0,1]$ were generated at $0.01,0.001$, and 0.0001 increments, etc. if necessary, and substituted into (9) until optimal solutions were found. The average solution time for each path-overlap model instance with $r=40.46$ feet in Xpress is 6.24 seconds. The tradeoff objectives are summarized visually in Figure 4.

Table 1 gives the lowest weights corresponding to unique objective values (3) and (4), representing the number of offices selected and path-overlap, respectively, for $r=40.46 \mathrm{feet}$. As an example, 


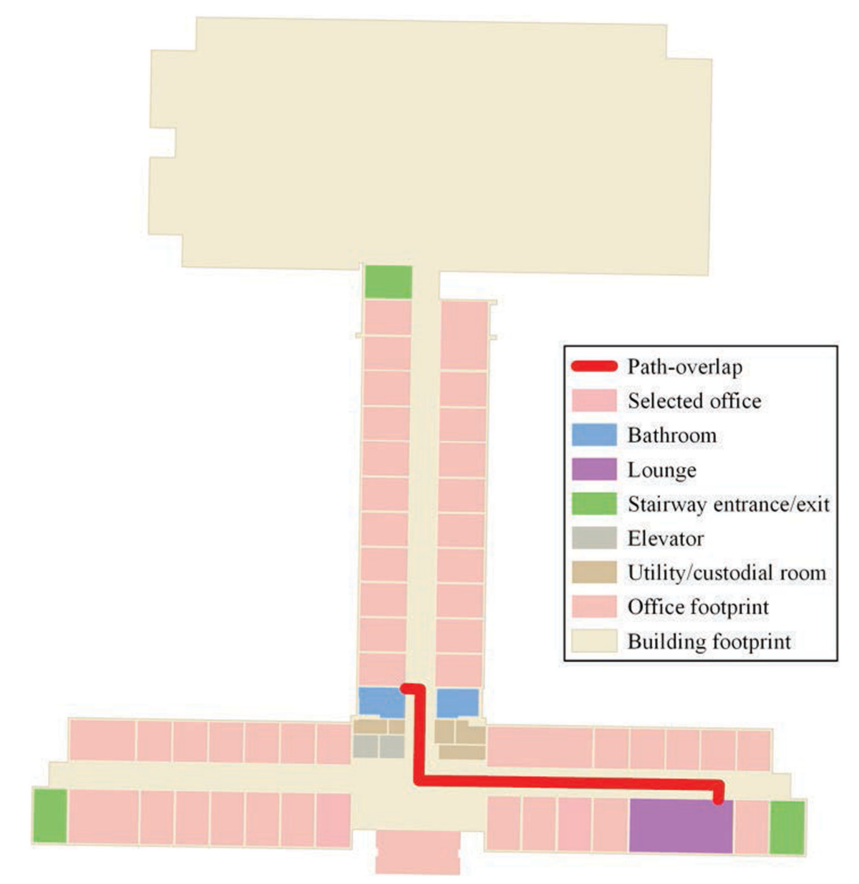

Fig. 5. Path-overlap for the two selected offices in Table $1(w=0.9930)$.

for $w=0.9930$ there are two offices selected and the resulting path-overlap is 124.91 feet. The offices and path-overlap corresponding to this solution are shown in Figure 5.

For the case of maintaining a separation distance of at least 40.36 feet, Table 1 indicates that a maximum of 7 offices could be sited $(w=0.9988)$, with a corresponding path-overlap of $2,707.65$ feet. This configuration of office occupancy is shown in Figure 6. It is not possible to accommodate more offices without violating the 40.36 feet separation requirement.

Of course, $r=40.36 \mathrm{ft}$. is a fairly restrictive separation distance. The building occupancy constraint of $15 \%$ strictly bounds what is possible in this scenario. However, it is possible to loosen this occupancy restriction through a change in the imposed separation distance. This is an important modeling feature and characteristic, enabling the path-overlap model to examine alternative scenarios with more or less restrictive interaction potential. Less spatial restriction may reflect later stages of the pandemic as more individuals become vaccinated, and the potential for disease spread decreases.

We demonstrate the richness of the path-overlap modeling approach with alternative scenarios and shorter $r$-separation distances. If the building occupancy limits are increased, then this would reflect less stringent office separation requirements. As an example, consider $r=20.12$ feet. The path-overlap model indicates an increased range of occupancy. The Pareto tradeoffs for this scenario are summarized in Table 2. The average solution time is 912.89 seconds (or 15.21 minutes). The maximum number of offices that can be sited in this scenario is 16 with the sum of overlapping paths equal to $16,132.34$ feet.

\section{DISCUSSION}

Office repopulation is a challenging task in the context of the COVID-19 pandemic. Supporting this planning and management effort through the use of the path-overlap model enables a 


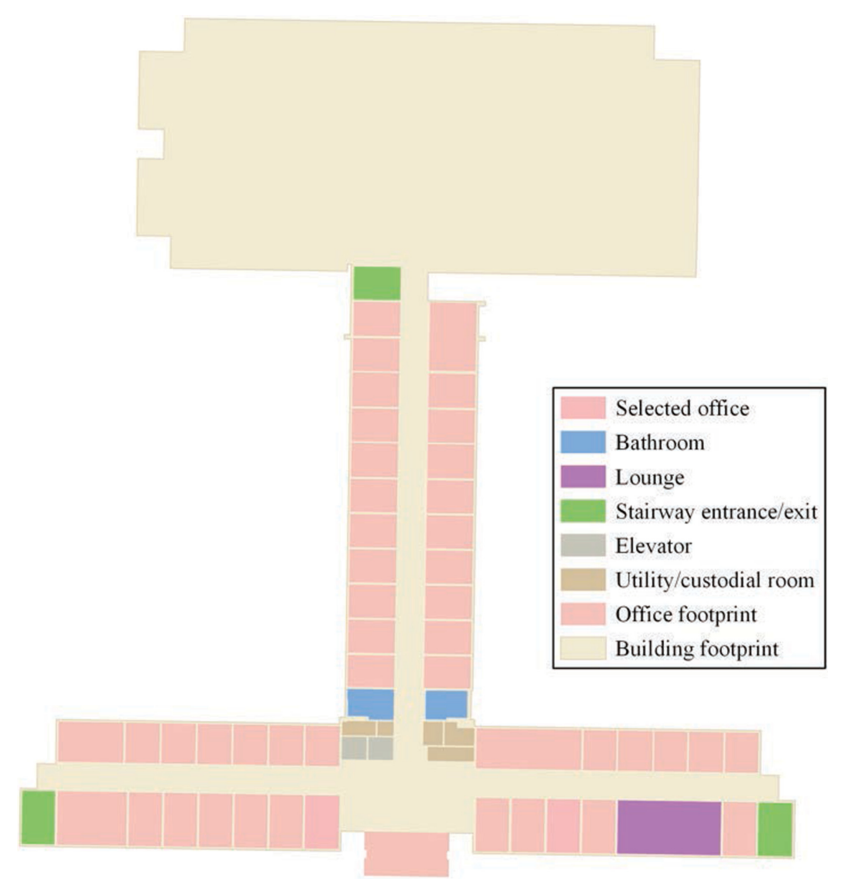

Fig. 6. Office configuration resulting for $r=40.36 \mathrm{ft}$. when $w=0.9930$.

Table 2. Pareto Tradeoffs for $r=20.12 \mathrm{ft}$

\begin{tabular}{lcc}
\hline $\boldsymbol{w}$ & Number of offices, (3) & Path-overlap, (4) \\
\hline 0.00100 & 1 & 0.00 \\
0.99300 & 2 & 124.91 \\
0.99700 & 3 & 374.73 \\
0.99800 & 4 & 749.47 \\
0.99802 & 5 & $1,249.12$ \\
0.99850 & 6 & $1,873.67$ \\
0.99880 & 7 & $2,629.05$ \\
0.99890 & 8 & $3,529.81$ \\
0.99910 & 9 & $4,560.11$ \\
0.99917 & 10 & $5,752.63$ \\
0.99930 & 11 & $7,074.09$ \\
0.99934 & 12 & $8,573.28$ \\
0.99939 & 13 & $10,202.48$ \\
0.99946 & 14 & $12,020.20$ \\
0.99950 & 15 & $13,976.60$ \\
0.99954 & 16 & $16,132.34$ \\
\hline
\end{tabular}

variety of scenarios to be considered. Varying the required separation distance along with consideration of potential for spatial interaction means that a variety of configurations for office repopulation are possible. Tables 1 and 2 show that siting a single office produces a trivial but validating case in which there are 0 feet of spatial interaction. Figures 4 and 7 demonstrate that as more 


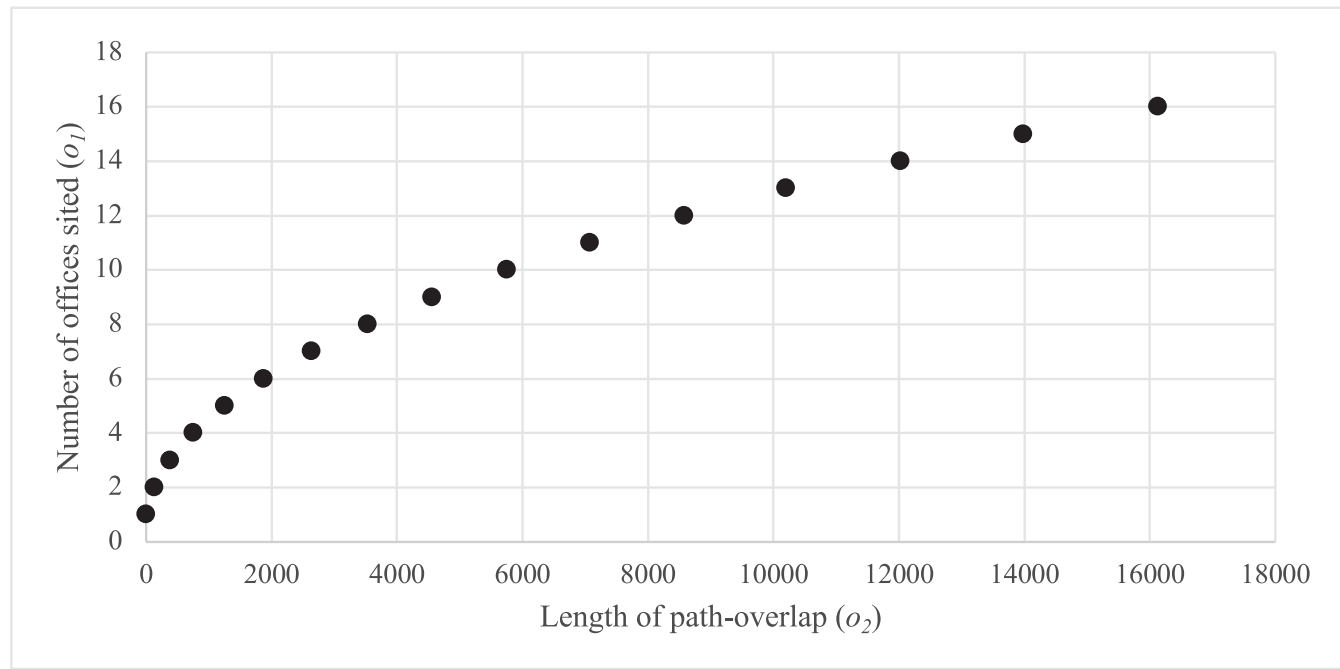

Fig. 7. Pareto tradeoffs for $r=20.12 \mathrm{ft}$.

offices are occupied, the amount of potential spatial interaction steadily increases as measured by path-overlap.

The spatial configuration shown in Figure 6 reveals interesting nuances of the planning problem, as well as capabilities of the proposed modeling approach. First, the model identifies configurations of dispersed offices as intended, siting mostly one or two offices for each hallway. The northern hallway (coinciding with the upper part of the floor) has three offices sited, which are as close to the bathrooms, lounge, and other hallways as possible. This reflects the intended result which is to reduce the length of the distance of path-overlap. Two offices in particular are directly next to or across from a bathroom or the lounge, limiting potential for interaction with other individuals en route to these facilities. These observations demonstrate that the model is effective at minimizing potential for interaction through the use of the path-overlap measure.

A second point worth mentioning is that as the allowable restriction distance decreases, more and more spatial configurations are possible as office occupancy can increase. These alternative configurations may actually reduce the potential for spatial interaction, or path-overlap. For example, when comparing Tables 1 and 2, the amount of path-overlap is the same for 1-4 offices sited. But for 5-7 offices sited the amount of path-overlap is actually higher for the $r$-separation distance of 40.36 feet. This is the case because some offices which would otherwise contribute to lower pathoverlap violate an $r$-separation distance with one another. So, while the $15 \%$ building occupancy constraint is being met in both scenarios of siting 5-7 offices, the model with the higher restriction distance performs more poorly than the model with the lower restriction distance in regard to potential interaction and path-overlap. When considering different alternatives, a decision-maker will have to evaluate these types of differences which are direct consequences of model constraints, such as if a restriction distance of 40.36 feet produces a meaningful advantage to 20.12 feet if the building occupancy requirement is otherwise being met. Furthermore, weights in the weighting method produce several different solutions. For example, when looking at Figure 6, it is apparent that an office even further above (closer towards the stairwell and away from the bathrooms) the most northern sited office could have been chosen, and the amount of path-overlap would have remained the same. Church and Baez [2020] explain why generating near-optimal solutions may be a preferred approach to many optimization problems, motivating the need to consider alternative solutions demonstrated here. 
Another interesting discussion item is the effect of even further reduction in physical distancing. Consider the case of a separation distance of at least 9 feet. The path-overlap model identifies the Pareto tradeoffs summarized in Table 3. Due to long solution times, the constraint method was applied, and most problems were cut off after 900 seconds (15 minutes) with the optimality gap (relative difference between the best known bound and the current objective value) reported. While optimality is not verified, these are likely optimal or close to optimal. Regardless, the maximum number of offices that can be sited at this restriction distance is 43 , with $122,891.18$ feet total overlapping paths. With a total of 46 offices, this many occupied offices on the fourth floor of Ellison Hall is quite high. This would result in the majority of neighboring offices being occupied, but is possible due to doorway to doorway distances of around 10 feet from one another. However, this type of scenario would be unacceptable under current pandemic conditions. It is thus interesting to note that the most restrictive $r$-separation distance of 40.36 feet that meets all of the spatial requirements (15\% building occupancy and having no offices sited adjacent from one another) is over six times greater than the United States Centers for Disease Control and Prevention's social distance requirement of 6 feet, and over four times greater than the university's preference of physical distancing of at least 9 feet. This indicates that perhaps the even more restrictive physical distance of 9 feet required by the university is inadequate alone as a measure for social distancing in a micro-spatial work environment, where prolonged periods of exposure and some movement is to be expected.

The path-overlap model, analyzed through various separation distances, reveals how planners, managers, and decision-makers for similar office environments can more generally account for different types of spatial structures and preferences. Our results show that, for this case study, a distance constraint, a proximity constraint, and a (building) occupancy constraint can all be addressed through parameterization ( $r$-separation distance), but that an interaction between building occupants must be addressed through an added objective (4). The GIS software used in this case study demonstrates that the addition of this second objective need not necessarily be technologically burdensome, as powerful GIS functionality is adept at quickly calculating topological operations and deriving the spatial detail necessary in optimization models.

There are some limitations to this work worth mentioning. The micro-spatial environment of one floor of a moderately-sized building made the weighting method for spatial optimization feasible, however, larger problems might need more advanced bi-objective solution techniques. While model solution times are not excessive, less restrictive distances also result in more challenging models to solve. This is because smaller $r$-separation distances produce fewer spatial restrictions, broaden the possibilities of alternative solutions, and force a longer exploration of the solution space (higher separation distances contribute to fewer restrictions on simultaneous selection and thus fewer constraints). The investigation of a separation distance of $r=9$ feet demonstrates how additional model formulation and solver tuning may be necessary to complete analyses in an appropriate time frame. Additionally, intensive time and effort was needed to digitize necessary building components and construct a walking path network, which would increase in complexity with the addition of multiple floors and mandated one-way flows. Despite this, a natural extension of this work would be to model an entire building with multiple floors. Furthermore, only the bathroom and lounge were considered accessible facilities in this case study, but other high traffic areas such as the stairway exit/entrance could readily be accommodated. The exit and entrance of a building in particular may present interesting spatial considerations, but since our case study draws from current building practices in which only one individual can be in a (closed) stairwell at a time, and stairwells connect floor entrances and exits from the building's outdoor entrances and exits, prohibiting close interaction, these considerations were not explored. Future work might address both a multifloor building and capacitated or one-way stairwells and hallways with a more 
Table 3. Pareto Tradeoffs for $r=9 \mathrm{ft}$

\begin{tabular}{|c|c|c|c|}
\hline Number of offices, (3) & Path-overlap, (4) & Solution time & Optimality gap (\%) \\
\hline 1 & 0.00 & 0.09 & 0.00 \\
\hline 2 & 124.91 & 0.11 & 0.00 \\
\hline 3 & 374.73 & 0.42 & 0.00 \\
\hline 4 & 749.47 & 3.22 & 0.00 \\
\hline 5 & $1,249.12$ & 11.58 & 0.00 \\
\hline 6 & $1,873.67$ & 63.94 & 0.00 \\
\hline 7 & $2,623.14$ & 242.15 & 0.00 \\
\hline 8 & $3,497.53$ & 900.34 & 3.40 \\
\hline 9 & $4,496.82$ & 900.11 & 20.93 \\
\hline 10 & $5,621.02$ & 900.14 & 21.10 \\
\hline 11 & $6,870.14$ & 900.12 & 23.32 \\
\hline 12 & $8,244.17$ & 900.10 & 27.83 \\
\hline 13 & $9,749.01$ & 900.11 & 32.21 \\
\hline 14 & $11,395.22$ & 900.09 & 34.43 \\
\hline 15 & $13,150.96$ & 900.12 & 33.50 \\
\hline 16 & $15,070.32$ & 900.10 & 34.49 \\
\hline 17 & $17,107.83$ & 900.13 & 32.57 \\
\hline 18 & $19,273.76$ & 900.11 & 29.06 \\
\hline 19 & $21,595.12$ & 900.11 & 33.04 \\
\hline 20 & $24,045.64$ & 900.13 & 28.33 \\
\hline 21 & $26,642.44$ & 900.14 & 22.56 \\
\hline 22 & $29,488.62$ & 900.13 & 28.03 \\
\hline 23 & $32,381.06$ & 900.09 & 23.74 \\
\hline 24 & $35,412.28$ & 900.13 & 21.54 \\
\hline 25 & $38,520.71$ & 900.11 & 19.00 \\
\hline 26 & $41,842.23$ & 900.10 & 15.20 \\
\hline 27 & $45,339.08$ & 900.11 & 11.84 \\
\hline 28 & $48,966.67$ & 900.11 & 9.88 \\
\hline 29 & $52,822.00$ & 900.10 & 6.63 \\
\hline 30 & $56,727.02$ & 900.09 & 4.45 \\
\hline 31 & $60,836.08$ & 900.10 & 3.10 \\
\hline 32 & $65,071.17$ & 755.37 & 0.00 \\
\hline 33 & $69,507.39$ & 766.58 & 0.00 \\
\hline 34 & $74,070.82$ & 127.94 & 0.00 \\
\hline 35 & $78,825.58$ & 48.31 & 0.00 \\
\hline 36 & $83,716.21$ & 20.99 & 0.00 \\
\hline 37 & $88,798.51$ & 10.45 & 0.00 \\
\hline 38 & $94,017.80$ & 2.79 & 0.00 \\
\hline 39 & $99,429.12$ & 1.26 & 0.00 \\
\hline 40 & $104,990.52$ & 0.65 & 0.00 \\
\hline 41 & $110,739.85$ & 0.45 & 0.00 \\
\hline 42 & $116,703.73$ & 0.39 & 0.00 \\
\hline 43 & $122,891.18$ & 0.16 & 0.00 \\
\hline
\end{tabular}


complicated network representation of the building. For example, there are many offices in which a single ventilation system, such as in a cubicle office setting, is a major impediment to more fully repopulating the workspace safely. In this scenario, constraints such as (5) and (6) will need to be modified to increase the separation distance until a safe density is reached. Otherwise, ventilation concerns must be met through more detailed modeling of ventilation zones, and in this scenario, the number of decision variables in constraints (5) and (6) must be increased to reflect these zones. Another important step in this analysis would include examining how office assignments change across time periods. For example, while the current results present optimal solutions for a subset of offices on the floor for one time period, there may be a planning scenario in which all individuals need equal access to their offices. Establishing cohorts of offices across multiple time periods in a day is an ambition for our case study's building management. Derived benefits of a given room $j, b_{j}$, could also impact spatial patterns and priorities. If it was deemed that certain individuals demonstrated a higher priority for obtaining access to their office (this might include critical staff, researchers with disruptive home environments, etc.), then $b_{j}$ could be scaled to reflect these prioritizations. None of these extensions would be prohibitively difficult to implement, and at the very least this work has provided a foundation for making such additions possible.

\section{CONCLUSIONS}

The leadership from public health institutions are imperative during a pandemic, but clearly operational decision-making needs guidance informed by location modeling. For example, our results show that a social distance of 9 (or even 6) feet would not likely be perceived as safe for certain office configurations as this would allow offices to be occupied that are next to one another and additionally, fails to adhere to strict occupancy building requirements. The path-overlap model structured as a bi-objective optimization problem reflects the important office repopulation goals in a meaningful manner, enabling an emphasis on potential interaction. Scenario evaluation and office occupancy configurations identified using the model improve the safety of a work environment.

During the pandemic, many hold the view that all safety precautions must be taken, as one minor interaction which results in a transmission and positive COVID-19 detection might endanger an individual and shut down an entire floor or building. Many universities and colleges must be extremely cautious during the pandemic given the greater density of student housing and the risk of higher spread in vibrant social networks, but the inevitably of interaction is not unique to educational settings. This work shows that prescriptive planning of office assignments can be applicable to a building or micro-spatial environment, and spatial optimization can identify alternatives that provide the greatest safety possible. Though present day is absorbed by the current pandemic, it is likely that even once everyone is vaccinated, extreme caution will still need to be used in office environments. Our methods and results offer guidance to all types of planning and decision-making where health concerns demand increased dispersion among individuals in shared spaces.

\section{REFERENCES}

L. Anselin and S. Bao. 1997. Exploratory spatial data analysis linking SpaceStat and ArcView. In Recent Developments in Spatial Analysis. Berlin: Springer, 35-59.

American Institute of Chemical Engineers. 2018. Guidelines for Siting and Layout of Facilities (Second ed.). Hoboken, NJ: Wiley.

W. E. Balson, J. L. Welsh, and D. S. Wilson. 1992. Using decision analysis and risk analysis to manage utility environmental risk. Interfaces 22, 6 (1992), 126-139.

Centers for Disease Control and Prevention. 2021. COVID-19: Prevent Getting Sick. Retrieved from https://www.cdc.gov/ coronavirus/2019-ncov/prevent-getting-sick/index.html.

R. L. Church and C. A. Baez. 2020. Generating optimal and near-optimal solutions to facility location problems. Environment and Planning B: Urban Analytics and City Science 47, 6 (2020), 1014-1030. 
R. L. Church and A. T. Murray. 2018. Location Covering Models: History, Applications and Advancements. Cham: Springer International Publishing.

R. L. Church and A. T. Murray. 2009. Business Site Selection, Location Analysis, and GIS. Hoboken, NJ: John Wiley \& Sons.

R. L. Church, A. T. Murray, and A. Weintraub. 1998. Locational issues in forest management. Location Science 6, 1-4 (1998), 137-153.

J. L. Cohon. 1978. Multiobjective Programming and Planning. New York: Academic Press.

P. W. Cunningham and A. Ellerbeck. 2021. When will 2021 feel normal again? Here's what eight experts predict. The Washington Post. Retrieved from https://www.washingtonpost.com.

J. A. Downs, R. J. Gates, and A. T. Murray. 2008. Estimating carrying capacity for sandhill cranes using habitat suitability and spatial optimization models. Ecological Modelling 214, 2-4 (2008), 284-292.

E. Erkut and S. Neuman. 1991. Comparison of four models for dispersing facilities. INFOR: Information Systems and Operational Research 29, 2 (1991), 68-86.

E. Erkut, C. Revelle, and Y. Ülküsal. 1996. Integer-friendly formulations for the r-separation problem. European fournal of Operational Research 92, 2 (1996), 342-351.

P. Fishburn. 1970. Utility Theory for Decision Making. New York: Wiley.

T. H. Grubesic and A. T. Murray. 2008. Sex offender residency and spatial equity. Applied Spatial Analysis and Policy 1, 3 (2008), 175-192.

M. Kuby. 1987. Programming models for facility dispersion: The p-dispersion and maxisum dispersion problems. Geographical Analysis 19, 4 (1987), 315-329.

J. Kudela. 2020. Social distancing as p-dispersion problem. IEEE Access 8, (2020), 149402-149411.

H. Kunreuther, K. Fitzgerald, and T. Aarts. 1993. Siting noxious facilities: A test of the facility siting credo. Risk Analysis 13, 3 (1993), 301-318.

D. G. McNeil. 2020. How can we achieve herd immunity? Experts are quietly upping the number. The New York Times. Retrieved from https://www.nytimes.com.

F. A. Medrano and R. L. Church. 2015. A parallel computing framework for finding the supported solutions to a biobjective network optimization problem. Journal of Multi-Criteria Decision Analysis 22, (5-6) (2015), 244-259.

R. Meller and K. Gau. 1996. The facility layout problem: Recent and emerging trends and perspectives. fournal of Manufacturing Systems 15, 5 (1996), 351-366.

I. D. Moon and S. S. Chaudhry. 1984. An analysis of network location problems with distance constraints. Management Science 30, 3 (1984), 290-307.

A. T. Murray. 1999. Spatial restrictions in harvest scheduling. Forest Science 45, 1 (1999), 45-52.

A. T. Murray. 2020. Planning for classroom physical distancing to minimize the threat of COVID-19 disease spread. PloS One 15, 12 (2020), e0243345.

A. T. Murray, R. L. Church, R. A. Gerrard, and W. S. Tsui. 1998. Impact models for siting undesirable facilities. Papers in Regional Science 77, 1 (1998), 19-36.

M. Newman. 2018. Networks: An Introduction. New York: Oxford University Press.

M. Niblett and R. Church. 2015. The disruptive anti-covering location problem. European fournal of Operational Research 247, 3 (2015), 764-773.

C. ReVelle. 1993. Facility siting and integer-friendly programming. European fournal of Operational Research 65, 2 (1993), 147-158.

L. Setti, F. Passarini, G. De Gennaro, P. Barbieri, M. G. Perrone, M. Borelli, J. Palmisani, A. Di Gilio, P. Piscitelli, and A. Miani. 2020. Airborne transmission route of COVID-19: Why 2 meters/6 feet of inter-personal distance could not be enough. International Journal of Environmental Research and Public Health 17, 8 (2020), 2932. https://doi.org/10.3390/ ijerph17082932.

R. Wei and A. T. Murray. 2018. Spatial uncertainty challenges in location modeling with dispersion requirements. In Spatial Analysis and Location Modeling in Urban and Regional Systems. Berlin: Springer, 283-300.

F. Zahedi. 1986. The analytic hierarchy process-A survey of the method and its applications. Interfaces 16, 4 (1986), 96-108.

R. E. Zeller, D. D. Achabal, and L. A. Brown. 1980. Market penetration and locational conflict in franchise systems. Decision Sciences 11, 1 (1980), 58-80.

Received April 2021; revised September 2021; accepted September 2021 